\title{
Multiple-State Quantum Carnot Engine
}

\author{
Eny Latifah, Agus Purwanto \\ Laboratory of Theoretical Physics and Natural Philosophy, Physics Department, \\ Institut Teknologi Sepuluh Nopember, Surabaya, Indonesia \\ E-mail: eny09@mhs.physics.its.ac.id,purwanto@physics.its.ac.id \\ Received August 27, 2011; revised September 30, 2011; accepted October 13, 2011
}

\begin{abstract}
A multiple-state quantum Carnot engine based on single particle in one dimensional potential well is evaluated. The general forms of adiabatic and isothermal force as well as work are given. We apply them first to the simplest case of two-state system, and then to three-state and general $n$-state system. The first isothermal expansion starts from single ground state and cease to single highest state. In Addition to the simplest case, isothermal expansions may terminate not to highest state but an intermediate state but with the same of the total expansion. The result is that the efficiency of the multi-state machine could be enhanced by reducing the volume of isothermal expansion for the same of the total volume expansion.
\end{abstract}

Keywords: Potential Well, Quantum Carnot Engine, Efficiency

\section{Introduction}

As a device to convert heat energy into mechanical work, classically, heat engine consists of gas as working agents that expands and pushes a piston in a cylinder. A heat engine obtains its energy from high temperature heat reservoir $T_{H}$. A part of this captured energy is converted into mechanical energy and the others are transferred to the low temperature reservoir $T_{C}$. Namely, not all of energy drawn from the reservoir $Q_{H}$ is converted into mechanical work $W$. Such that the efficiency of the machine, which is defined as the ratio between mechanical work $W$ and energy absorbed by the machine $Q_{H}$, will be less than one

$$
\eta=\frac{W}{Q_{H}}<1 .
$$

The main problem of a heat engine is none other than this efficiency which is generally small or low. Heat engine, working between high temperature reservoir and low temperature reservoir, will reach maximum efficiency if the process is reversible.

Mathematical model of an ideal heat engine was proposed by Sadi Carnot in 1824. It is reversible and has the highest possible efficiency for any engine operating between two given temperatures. For this reason, it serves as a model for real engines to emulate [1]. Carnot's engine is constructed by a cylinder containing an ideal gas placed in a thermal contact with high and low reser- voirs temperatures, alternately. It consists of four processes, two isothermal and two adiabatic processes, each of which is reversible. First, the gas in the cylinder undergoes an isothermal expansion at temperature $T_{H}$ while it is in contact with the high-temperature reservoir. Second, the gas continues to expand adiabatically in thermal isolation until its temperature drops to $T_{C}$. Third, the gas is compressed isothermally in contact with the low temperature reservoir. Fourth, the gas is compressed adiabatically until its temperature rises to $T_{H}$. As mentioned before, the interesting problem is the low efficiency of the engine.

One of the efforts to increase the efficiency of heat engine the application of quantum principles to the engine. The study of quantum engines started in 60's [2], when it was realized that many models of lasers and masers are in fact quantum heat engines. A good review of this early activity is given by [3] in [4]. Nowadays the physics of quantum heat engines is a rich field, some papers describe one by studying the quantum thermodynamical processes, quantum generalization of force or preasure to study quantum version of thermodynamic cycles [5-8]. To enhance the efficiency of heat engines, a paper applied the principles of quantum mechanics to the heat engine. It had been efforted using a laser and masser in tandem. As an example, it is shown that the performance of Otto cycle engine can be improved beyond that of the "ideal" Otto heat engine [9]. While, other author stated that the quantum efficiency can exceed the classi- 
cal Carnot limit with quantum correlation [10].

Bender, et al., [11] provided a kind of cyclic Carnot heat engine employing a single quantum-mechanical particle, as a working substance, confined to a potential well, instead of gas-filled cylinder. The cycle consists of isothermal and adiabatic quantum processes that are close analogues of the corresponding classical processes. By formulating 2-state quantum heat engine the efficiency of this system is analogue of the classical thermodynamical result of Carnot. It would be more interesting if it is studied for $n$-state in greater depth. In other hand, by reformulating the first law of thermodynamics in the fashion of quantum-mechanical operator on the parameter manifold, Quan et al. proposed a class of quantum heat engines using the multilevel quantum system as the working substance. They proved for a 3-level QHE the work to be looser than that for a 2-level system under certain conditions [5].

The purpose of this paper is further discussion of quantum Carnot heat engine employing a single quantum-mechanical particle confined to a potential well with a multiplestate particle. In previous work [11], the results of 2-state was generalized to $n$-state qualitatively without detail analysis. Since the particle system has multistate then it has some eigenstates, at least one state, before the highest state which can be reached as a final state in isothermal expansion, this fact in turn gives a possibility to choose another final state besides the highest eigenstate of the system. For the same total expansion but different expansion during isothermal process gives a different efficiency.

This paper is organized as follows: In Section 2 we briefly review the simplest quantum mechanical Carnot engine based on one-dimensional potential well with two eigenstates. In Section 3, three level and generalization to $n$-level quantum system is discussed. Finally, discussion and conclusions will be given in Section 4 .

\section{Quantum Carnot Engine}

Quantum heat engines (QHEs) [2] produce work using quantum matter as their working substance. Because of the quantum nature of the working substance, QHE have unusual and exotic properties. For example, under some conditions, QHEs can surpass the maximum limit on the amount of work done by a classical thermodynamic cycle [12] and also surpass the efficiency of a classical Carnot engine cycle. QHEs offer good model systems to study the relation between thermodynamics and quantum mechanics. Meanwhile, they can highlight the difference between classical and quantum thermodynamic systems, and help us understand the quantum classical transition problem of thermodynamic processes [6,7].
We provided a kind of quantum heat engines, i.e. a cyclic Carnot heat engine employing a single quantummechanical particle, as a working substance, confined to a potential well, instead of gas-filled cylinder. Rather than 2-state, we purpose further discuccion with 3-state and $n$-state of the particle.

\subsection{Infinite One-Dimensional Potential Well}

The simplest quantum system is a particle mass $m$ confined in a one-dimensional well of width $L$ with infinite potential walls. The motion equation of this system is one-dimensional time independent Schrodinger equation.

$$
-\frac{\hbar^{2}}{2 m} \frac{d^{2} \varphi}{d x^{2}}=E \varphi .
$$

The infinite potential walls at $x=0$ and $x=L$ provide boundary conditions for the state functions $\varphi(0)=\varphi(L)=$ 0 . The general solution of this equation is a linear combination of orthonormal eigenfunctions $\varphi_{n}(x)$

$$
\varphi(x)=\sum_{n=1}^{\infty} a_{n} \varphi_{n}(x)
$$

and energy $E$,

$$
E=\sum_{n=1}^{\infty}\left|a_{n}\right|^{2} E_{n},
$$

where $\varphi_{n}$ and $E_{n}$ have explicit forms as follows.

$$
\begin{aligned}
\varphi_{n}(x) & =\sqrt{\frac{2}{L}} \sin \left(\frac{n \pi}{L} x\right), \\
E_{n} & =n^{2} \frac{\pi^{2} \hbar^{2}}{2 m L^{2}} .
\end{aligned}
$$

And, the coefficients $a_{n}$ satisfy the orthonormal condition

$$
\sum_{n=1}^{\infty}\left|a_{n}\right|^{2}=1,
$$

From Equation (6), the energy (4) becomes

$$
E=\sum_{n=1}^{\infty}\left\{n^{2}\left|a_{n}\right|^{2}\right\} \frac{\pi^{2} \hbar^{2}}{2 m L^{2}} .
$$

Now, if one of the infinite walls of the potential well, say the wall at $x=L$, is allowed to move an infinitesimal amount $d L$ then the general wave function $\varphi(x)$, eigen functions $\varphi_{n}(x)$ and energy levels $E_{\mathrm{n}}$ all vary infinitesimally as function of $L$. In this situation, it is natural to define the force on the wall of the potential well as the negative derivative of the energy,

$$
F=-\frac{d E}{d L} .
$$


This force $F$ exerts on the wall. Based on this force, several kinds of thermodynamical processes which have the quantum analogues to the classics can be defined. According to the force, work from $L_{1}$ to $L_{2}$ is given by

$$
W_{12}=\int_{L_{1}}^{L_{2}} F \mathrm{~d} L .
$$

\subsection{Thermodynamical Quantum Processes}

Heat engines are devices that extract energy from its environment in the form of heat and do useful work. The heart of every heat engine is its working substance. The operation of the heat engine is by subjecting the working substance of the engine to be a sequence of thermodynamics processes that forms a cycle. Carnot heat engine operates with isothermal and adiabatic processes. Classically, an adiabatic process is one in which the system is thermally isolated in such a way that heat cannot flow into or out of the system. An isothermal process is one in which as the piston moves, the system remain in equilibrium at all times. During the process, the system is in contact with a heat reservoir so that the temperature $T$ of the gas in cylinder remains fixed. During the piston moves, the system does work both in adiabatic as well as in isothermal processes.

Now, the above classical thermodynamical system may be applied in a monatomic one-dimensional gas in the infinite potential well. One monatomic particle is as a working substance, a wall of infinite potential well at $x=$ $L$ as a piston can moves and the system remains in equilibrium at all times. Two processes for Carnot engine are adiabatic and isothermal.

In adiabatic process, there is no heat transfer from or into the system, and the potential wall changes as the wall moves. Since the system remains in equilibrium at all times, the absolute values of the expansion coefficients $\left|a_{n}\right|$ must be constant. That is, we do not expect any transitions between states can occur during an adiabatic process.

It is clear from Equation (7) that the eigen energy $E_{n}$ depends on $L$ and the energy (4) will changes during an adiabatic process. The energy increases if the system compresses and decreases if the system expands. When the piston moves out, then the energy decreases and the energy that is lost equals the mechanical work done by the force $F(9)$, which is given by

$$
F=\left\{\sum_{n=1}^{\infty} n^{2}\left|a_{n}\left(L_{i}\right)\right|^{2}\right\} \frac{\pi^{2} \hbar^{2}}{m L^{3}},
$$

where $a_{n}\left(L_{\mathrm{i}}\right)$ is the $n$-th coefficicnt at initial state with a width $L_{\mathrm{i}}$.

Applying the force (11) into Equation (10), we obtain a work along adiabatic process from $L_{1}$ as the initial volume to $L_{2}$ as the final volume

$$
W_{12}=\left\{\sum_{n=1}^{\infty} n^{2}\left|a_{n}\left(L_{1}\right)\right|^{2}\right\} \frac{\pi^{2} \hbar^{2}}{2 m}\left(\frac{1}{L_{1}^{2}}-\frac{1}{L_{2}^{2}}\right) .
$$

In isothermal process, the system is in contact with a heat source so that the temperature $T$ of the gas in the cylinder is fixed. It implies that the transition between states occur so that the internal energy of the gas during isothermal process remains constant. In other word, the expansion coefficients $a_{n}$ in general change but the orthonormal condition (5) remains satisfied. This implies that during the process the width of the system satisfies

$$
L^{2}=\frac{\sum_{n=1}^{\infty} n^{2}\left|a_{n}(L)\right|^{2}}{\sum_{n=1}^{\infty} n^{2}\left|a_{n}\left(L_{i}\right)\right|^{2}} L_{i}^{2} .
$$

Where $L_{\mathrm{i}}$ is the initial width.

The force of the piston is constrained by Equation (12) and has a form as follows.

$$
F=\left\{\sum_{n=1}^{\infty} n^{2}\left|a_{n}\left(L_{i}\right)\right|^{2}\right\} \frac{\pi^{2} \hbar^{2}}{m L_{i}^{2} L} .
$$

According to the force, the work along isothermal process from $L_{1}$ as the initial volume, to $L_{2}$ as the final volume is given by

$$
W_{12}=\left\{\sum_{n=1}^{\infty} n^{2}\left|a_{n}\left(L_{1}\right)\right|^{2}\right\} \frac{\pi^{2} \hbar^{2}}{m L_{1}^{2}} \ln \left(\frac{L_{2}}{L_{1}}\right) .
$$

A work of the system is equal to the absorbed energy.

\subsection{Two-State Quantum Carnot Engine}

Using the two quantum adiabatic processes and two quantum isothermal processes above, we can construct a Carnot heat engine. The simplest case of the particle in the system is the particle has only two states i.e. ground state $(n=1)$ and the first excited state $(n=2)$. It means the orthonormal condition (5) becomes simpler form $\left|a_{1}\right|^{2}+\left|a_{2}\right|^{2}=1$.

The four-step cyclic quantum process of the quantum Carnot engine is illustrated as a diagram between the force and the width as in Figure 1. The cycle starts with a ground state in a well of width $L_{\mathrm{A}}$. It means the coefficients $a_{1}=1$ and $a_{2}=0$. The energy of the system is the lowest value. The wall at $L_{\mathrm{A}}$ expands isothermally and the particle may excite and in general the state of particle is combination of two possible states $\varphi=a_{1} \varphi_{1}+a_{2} \varphi_{2}$. Along the expansion, the relation of widths (13) has a simple form 


$$
L^{2}=\left(a_{1}^{2}+4 a_{2}^{2}\right) L_{A}^{2} .
$$

It gives, in turn, the maximum expansion $L_{B}=2 L_{\mathrm{A}}$ when the state of particle is purely excited state $a_{2}=1$ and $a_{1}=0$. If the expansion is maximum then the total work, that is the work done to expand from $L_{A}$ to $2 L_{A}$ is given by

$$
W_{A B}=\frac{\pi^{2} \hbar^{2}}{m L_{A}^{2}} \ln 2 .
$$

The second expansion from $L_{B}$ is adiabatic expansion with a single state $\varphi_{2}$. Different from isothermal process, there is no definit limit of the maximum width in the adiabatic process. Then we assume the piston moves from $L_{B}$ to $L_{C}=\alpha L_{B}$ where the real number $\alpha$ is bigger than one, then the work during the isothermal expansion is given by

$$
W_{B C}=4 \frac{\pi^{2} \hbar^{2}}{2 m L_{B}^{2}}\left(1-\frac{1}{\alpha^{2}}\right) .
$$

Next, the system is compressed isothermally from $L_{C}$ to $L_{D}$. At the beginning of compression the system is purely in the first excited state, $a_{1}=0$ and $a_{2}=1$. Along the compression the transistion occurs, the system is in the mixed state and the energy remains constant. In order the process occurs in close loop then at width $L_{D}$ the system should be at a single ground state. It means $a_{1}\left(L_{D}\right)=$ $1, a_{2}\left(L_{D}\right)=0$. Since $a_{1}\left(L_{C}\right)=0, a_{2}\left(L_{C}\right)=1$ then from (13) we obtain a maximum compression $L_{D}=L_{C} / 2$. Hence, the isothermal work during compression from $L_{C}$ to $L_{D}=L_{C} / 2$ is given by

$$
W_{C D}=-4 \frac{\pi^{2} \hbar^{2}}{m L_{C}^{2}} \ln 2 .
$$

The last process is adiabatic compression from $L_{D}$ to $L_{A}$. During the compression the system is in single ground state. The work of the compression is given by

$$
W_{D A}=\frac{\pi^{2} \hbar^{2}}{2 m}\left(\frac{1}{L_{D}^{2}}-\frac{1}{L_{A}^{2}}\right) .
$$

The mechanical work $W$ done in single cycle of the quantum heat engine is represented by the area of the closed loop in Figure 1. Using relation between $L_{B}=2 L_{A}$, $L_{C}=\alpha L_{B}$ and $L_{D}=L_{C} / 2$ the total mechanical work is the sum of $W_{A B}, W_{B C}, W_{C D}$, and $W_{D A}$. The total work is

$$
W=\frac{\pi^{2} \hbar^{2}}{m L_{A}^{2}} \ln 2\left(1-\frac{1}{\alpha^{2}}\right),
$$

and the efficiency is the ratio between $W$ and $W_{A B}$ and given by

$$
\eta=1-\frac{1}{\alpha^{2}} .
$$

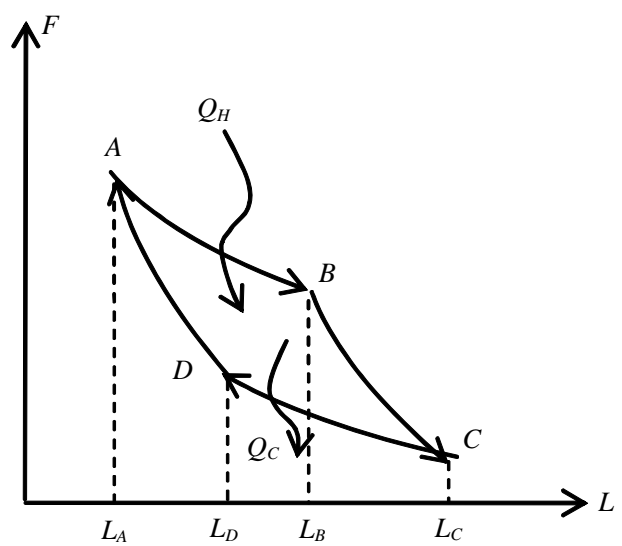

Figure 1. Cycle of carnot engine.

\section{Multiple-State}

Formulation can be generalized, as stated qualitatively by Bender [11], to be $n$-state and if the initial width of the wall is $L_{1}$ then the maximum expansion is $n L_{1}$. We study more detail for the case of $n$-state, and first study for 3-state.

\subsection{3-State}

We assume the system may have three eigen states $\varphi_{1}, \varphi_{2}$, $\varphi_{3}$ associated with the eigen energies $E_{1}, E_{2}$ and $E_{3}$. As in case of two-state, we start with the initial state a ground state $\varphi_{1}$ with energy $E_{1}$ of width $L_{A}$. The expansion coefficiens of the initial condition are $a_{1}=1, a_{2}=a_{3}=0$. Then, the system isothermally expands and does transition to all three possible states. In general the system is in a linear combination of three states $\varphi=a_{1} \varphi_{1}+a_{2} \varphi_{2}+$ $a_{3} \varphi_{3}$ and satisfies orthonormal condition

$$
\left|a_{1}(L)\right|^{2}+\left|a_{2}(L)\right|^{2}+\left|a_{3}(L)\right|^{2}=1 .
$$

Using $a_{1}(L)=1, a_{2}(L)=a_{3}(L)=0$, Equation (13) becomes

$$
L^{2}=\left(\left|a_{1}\right|^{2}+4\left|a_{2}\right|^{2}+9\left|a_{3}\right|^{2}\right) L_{A}^{2},
$$

yielding maximum expansion $L=L_{\mathrm{C}}=3 L_{\mathrm{A}}$ which is occurred when $a_{1}\left(L_{B}\right)=a_{2}\left(L_{B}\right)=0$ and $a_{3}\left(L_{B}\right)=1$. Hence, the work until maximum expansion is given by

$$
W_{A B}=\frac{\pi^{2} \hbar^{2}}{m L_{A}^{2}} \ln 3 .
$$

The second expansion from $L_{B}$ to $L_{B}=\alpha L_{C}$ occurs in highest state $\varphi_{3}$. The adiabatic work (12) becomes

$$
W_{B C}=9 \frac{\pi^{2} \hbar^{2}}{2 m L_{B}^{2}}\left(1-\frac{1}{\alpha^{2}}\right) .
$$

For the isothermal compression from $L_{C}$ to $L_{D}$ the ini- 
tial conditions of the system are $a_{1}=a_{2}=0$ and $a_{3}=1$ and then does transition to the linear combination of three possible states. Relation (13) reduces to the form

$$
L^{2}=\frac{\left|a_{1}\right|^{2}+4\left|a_{2}\right|^{2}+9\left|a_{3}\right|^{2}}{9} L_{C}^{2},
$$

yielding a maximum compression to $L_{D}=L_{C} / 3$ with the system in a single ground state. The associated work to maximum compression is given by

$$
W_{C D}=-9 \frac{\pi^{2} \hbar^{2}}{m L_{C}^{2}} \ln 3 .
$$

From relation among $L_{A}, L_{B}, L_{C}$ and $L_{D}$ we obtain work of the adiabatic compression in the form of initial width $L_{A}$ of the whole process

$$
W_{D A}=-\frac{\pi^{2} \hbar^{2}}{2 m L_{A}^{2}}\left(1-\frac{1}{\alpha^{2}}\right) .
$$

Then, the total work is given by

$$
W=\frac{\pi^{2} \hbar^{2}}{m L_{A}^{2}} \ln 3\left(1-\frac{1}{\alpha^{2}}\right),
$$

and the efficiency is the same with a case of two states

$$
\eta=1-\frac{1}{\alpha^{2}} \text {. }
$$

We are also interested in other case. We consider first the multiply factor $\alpha$ that is the same for two cases, two-states and three-states, $\alpha=L_{C} / L_{B}$. But, two previous cases are different at total expansion from initial width, $2 \alpha$ for two-states and $3 \alpha$ for three-states.

Now we consider a case of the expansion from initial witdh $L_{A}$ not to $L_{B}=3 L_{A}$ but to $L_{B}=2 L_{A}$. When the system expands isothermally and comes to rest at $L_{B}=2 L_{A}$ then the work of the system is

$$
W_{A B}=\frac{\pi^{2} \hbar^{2}}{m L_{A}^{2}} \ln 2 .
$$

At $L_{B}=2 L_{A}$ the system still has a linear combination of all three possible states, and the coefficients $a_{1}, a_{2}$ and $a_{3}$ satisfy a relation

$$
\left|a_{1}\right|^{2}+4\left|a_{2}\right|^{2}+9\left|a_{3}\right|^{2}=4 .
$$

Equation (30) and orthonormal condition (22) form a line of condition of the system during adiabatic expansion from $L_{B}=2 L_{A}$ to $L_{C}$ (Figure 2).

When the system expands from $2 L_{A}$ to $L_{C}$, its state is a fixed definite state at a line state Figure 2. For $L_{C}=3 \alpha L_{A}$ then the work (13) becomes

$$
W_{B C}=\frac{\pi^{2} \hbar^{2}}{2 m L_{A}^{2}}\left(1-\frac{4}{9} \frac{1}{\alpha^{2}}\right) .
$$

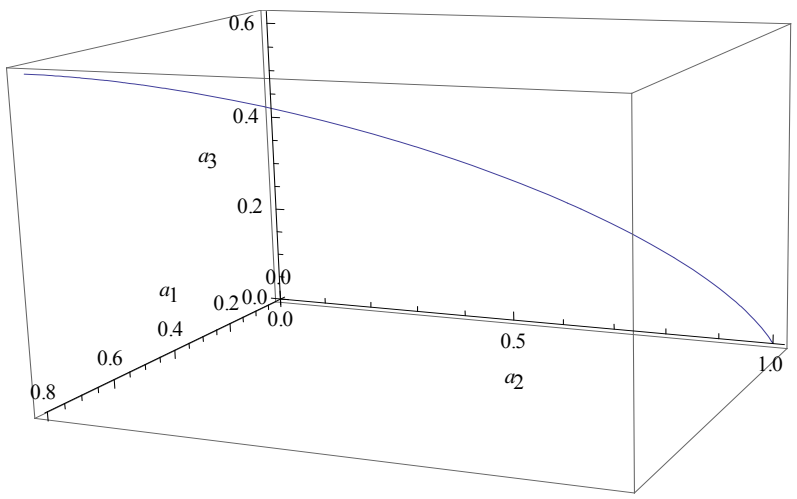

Figure 2. Parameters $\left|a_{1}\right|,\left|a_{2}\right|$ and $\left|a_{3}\right|$ along the adiabatic expansion.

The next process is isothermal compression from $L_{C}=$ $3 \alpha L_{A}$ to $L_{D}$. Since the expansion from $L_{B}$ to $L_{C}$ is adiabatic then the coefficients do not change. It implies that condition (30) prevails also at $L_{C}$. It further implies, from relation (13) that the maximum compression from $L_{C}$ is is $L_{D}=L_{C} / 2$, and the work is given by

$$
W_{C D}=-\frac{\pi^{2} \hbar^{2}}{m L_{A}^{2}}\left(\frac{4}{9} \frac{1}{\alpha^{2}}\right) \ln 2 .
$$

At wall of width $L_{C} / 2$ the system is at ground state, and then expands adiabatically until $L_{A}$. Since $L_{D}=$ $3 \alpha L_{A} / 2$ then its work of the compression from $L_{D}$ to $L_{A}$ is

$$
W_{D A}=-\frac{\pi^{2} \hbar^{2}}{2 m L_{A}^{2}}\left(1-\frac{4}{9} \frac{1}{\alpha^{2}}\right) \text {. }
$$

The total work of the cycle is

$$
W=\frac{\pi^{2} \hbar^{2}}{m L_{A}^{2}}\left(1-\frac{4}{9} \frac{1}{\alpha^{2}}\right) \ln 2,
$$

and the efficiency of the heat engine $W / W_{A B}$ is given by

$$
\eta=1-\frac{4}{9} \frac{1}{\alpha^{2}}
$$

\subsection{General Equations}

It is straightforward to generalize the three-state quantum Carnot engine to $n$-state. For $n$-state with initial state is single ground state and width is $L_{A}$, the isothermal expansion can be maximum of width $n L_{A}$. During the expansion the system does transition to all possible $n$ states and it is as a liniear combination of all $n$-state. The coefficients of the expansion satisfy an orthonormal condition

$$
\left|a_{1}\right|^{2}+\left|a_{2}\right|^{2}+\cdots+\left|a_{n}\right|^{2}=1 .
$$

At the maximum width the system back to a single state, the highest excited state, $n^{\text {th }}$-state. The work of the 
expansion is

$$
W_{1}=\frac{\pi^{2} \hbar^{2}}{m L_{A}^{2}} \ln n .
$$

The next expansion is adiabatic expansion from $n L_{A}$ to $n \alpha L_{A}$. During the expansion, the state is in single $n$-state. The work is

$$
W_{2}=\frac{\pi^{2} \hbar^{2}}{2 m L_{A}^{2}}\left(1-\frac{1}{\alpha^{2}}\right),
$$

that depend on the ratio of final and initial width of the adiabatic expansion.

The isothermal compression occurs with condition (13) of the form

$$
L^{2}=\frac{\left|a_{1}\right|^{2}+4\left|a_{2}\right|^{2}+\cdots+n^{2}\left|a_{n}\right|^{2}}{n^{2}} L_{C}^{2},
$$

then the maximum compression that leads to cyclic process is $L_{D}=L_{C} / n$. The work is

$$
W_{3}=-\frac{\pi^{2} \hbar^{2}}{m \alpha^{2} L_{A}^{2}} \ln n .
$$

The adiabatic compression occurs in single ground state and its work is negative of adiabatic expansion (38). Then the total of cyclic work is

$$
W=\frac{\pi^{2} \hbar^{2}}{m L_{A}^{2}} \ln n\left(1-\frac{1}{\alpha^{2}}\right),
$$

with the efficiency is equal to efficiency of 2 or 3 states (28).

The second scenario of system with $n$ states is the isothermal expansion not to maximum $n L_{A}$ but $k L_{A}$ where integer $k$ is smaller than $n$. When the system expands isothermally and comes to rest at $L_{B}=k L_{A}$ then the work of the system is

$$
W_{1}=\frac{\pi^{2} \hbar^{2}}{m L_{A}^{2}} \ln k
$$

At $L_{B}=k L_{A}$ the system still has a linear combination of all possible states, and the coefficients $a_{1}, a_{2}, \cdots, a_{n}$ also satisfy a relation

$$
\left|a_{1}\right|^{2}+4\left|a_{2}\right|^{2}+\cdots+n^{2}\left|a_{n}\right|^{2}=k^{2} .
$$

Equation (42) and orthonormal condition (36) make intersection and give a condition of the system along adiabatic expansion from $L_{B}=k L_{A}$ to $L_{C}$.

When the system expands from $k L_{A}$ to $L_{C}$ its state is a fixed definite state at a point at hypervolume of intersecttion of orthonormal condition (36) and coefficient of maximum expansion. The coefficient number of force is given condition (42) and expansion from $L_{B}=k L_{A}$ to $L_{C}$ $=n \alpha L_{A}$ is performed by the work

$$
W_{2}=\frac{\pi^{2} \hbar^{2}}{2 m L_{A}^{2}}\left(1-\frac{k^{2}}{n^{2}} \frac{1}{\alpha^{2}}\right) .
$$

The next process is isothermal compression, with the initial width $L_{C}=n \alpha L_{A}$. Condition (42) yields a maximum compression $L_{D}=L_{C} / k$ where at this width the system is at single a ground state. The work is given by

$$
W_{3}=-\frac{\pi^{2} \hbar^{2}}{m L_{A}^{2}}\left(\frac{k^{2}}{n^{2}} \frac{1}{\alpha^{2}}\right) \ln k .
$$

At wall of width $L_{C} / k$ the system is at ground state, and then expands adiabatically until $L_{A}$. The work of compression is given by

$$
W_{4}=-\frac{\pi^{2} \hbar^{2}}{2 m L_{A}^{2}}\left(1-\frac{k^{2}}{n^{2}} \frac{1}{\alpha^{2}}\right) .
$$

The total work of the cycle is

$$
W=\frac{\pi^{2} \hbar^{2}}{m L_{A}^{2}}\left(1-\frac{k^{2}}{n^{2} \alpha^{2}}\right) \ln k,
$$

and the efficiency of the heat engine $W / W_{A B}$ depends on the first expansion factor $k$ and total expansion $n \alpha$

$$
\eta=1-\frac{k^{2}}{n^{2} \alpha^{2}} .
$$

In the perspective of energy, since $L_{C}=\alpha L_{B}$, then the efficiency (28) which is also satisfied by system of $n$ state is the same to

$$
\eta=1-\frac{E_{B}}{E_{C}} .
$$

Whereas, the efficiency (47) for the total expansion $n \alpha L_{A}$ but intermediate expansion $k L_{A}$ is the same to

$$
\eta=1-\frac{E_{B}^{\prime}}{E_{C}},
$$

where $E_{B}^{\prime}=\left(k^{2} / n^{2}\right) E_{B}$.

\section{Conclusions}

We have elaborated the quantum mechanical Carnot engine [11]. The general result is the same as the previous work, that is the efficiency depends on the initial and final energy or temperature [11]. This result makes a general confirmation that for the higher state QHE the work to be looser than than that for lower sate system under certain condition [5]. However, there is more interesting result in the perspective of the volume of system. In the perspective energy or temperature, the efficiency may be increased if the lower energy is descended. It may be performed by, for the same total expansion, lowering of the isothermal expansion. The later lower 
energy is smaller than the former by a factor $k^{2} / n^{2}$. In other statement, if the too many number of state of system, causing decreasing efficiency, cannot be control, the efficiency can be enhanced by controlling its isothermal expansion.

The maximum isothermal expansion $L_{B}$ of the $n$ states system is $n L_{A}$. It is occurred when both initial and final state are single state, ground state for initial state and the highest state for the final state. However, if the initial width in the state of $k$ then the maximum expansion is $n L_{A} / k$.

\section{Acknowledgements}

The authors give thank to the Theoretical Physics Group at University of Indonesia for its hospitality during Conference on Theoretical Physics and Nonlinoear Phenomena 2010. This work was support of Indonesia Ministry of Education.

\section{References}

[1] K. Stowe, "An Introduction to Thermodynamics and Statistical Mechanics," 2nd Edition, Cambridge University, Cambridge, 2007.

[2] H. E. D. Scovil and E. O. Schul-DuBois, "Three-Level Masers as Heat Engines," Physical Review Letters, Vol. 2, No. 6, 1959, pp. 262-263.

doi:10.1103/PhysRevLett.2.262

[3] В. K. Konyukhov and A. M. Prokhorov, "Второе начало термодинамики и квантовые генераторы с тепловым возбуждением," UspekhiFiz. Nauk, Vol. 119, 1976, pp.
541-550. doi:10.3367/UFNr.0119.197607f.0541

[4] A. E. Allahverdyan, R. S. Johal and G. Mahler, "Work Extremum Principle: Structure and Function of Quantum Heat Engines," 2007, arXiv: 0709.4125v1.

[5] H. T. Quan, P. Zhang and C. P. Sun, "Quantum Heat Engine with Multi-Level Quantum Systems," Physical Review E, Vol. 72, No. 5, 2005, p. 056110. doi:10.1103/PhysRevE.72.056110

[6] H. T. Quan, P. Zhang and C. P. Sun, "Quantum-Classical Transition of Photon-Carnot Engine Induced by Quantum Decoherence," Physical Review E, Vol. 73, No. 3, 2006 , p. 036122.doi:10.1103/PhysRevE.73.036122

[7] H. T. Quan, Y.-X., Liu, C. P. Sun and F. Nori, "Quantum Thermodynamic Cycles and Quantum Heat Engines," Physical Review E, Vol. 76, No. 3, 2007, p. 031105. doi:10.1103/PhysRevE.76.031105

[8] H. T. Quan, "Quantum Thermodynamic Cycles and Quantum Heat Engines (II)," Physical Review E, Vol. 79, No. 4, 2009, p. 041129.doi:10.1103/PhysRevE.79.041129

[9] M. O. Scully, "The Quantum Afterburner: Improving the Efficiency of an Ideal Heat Engine," Physical Review Letters, Vol. 88, No. 5, 2002, p. 050602 doi:10.1103/PhysRevLett.88.050602

[10] R. Dillenschneider and E. Lutz, "Improving Quantum Carnot Engine with Quantum Correlation," 2008. arXiv:0803.4067v1.

[11] C. M. Bender, D. C. Broody and B. K. Meisner, "Quantum Mechanical Carnot Engine," Journal of Physics, Vol. 33, No. 24, 2000, p. 4427.

[12] T. D. Kieu, "Quantum Heat Engines, The Second Law and Maxwell's Demon," European Physical Journal D, Vol. 39, No. 1, 2006, pp. 115-128. doi:10.1140/epjd/e2006-00075-5 